\title{
TRANSLATING ONTOLOGIES TO DEFAULT LOGIC
}

\author{
Yu Sun ${ }^{1}$ and Yuefei Sui ${ }^{2}$ \\ ${ }_{1,2}$ Key Laboratory of Intelligent Information Processing, Institute of Computing Technology, \\ Chinese Academy of Sciences, Beijing, 100080, P.R.China; 'Institute of Computer Science and \\ Information Technology, Yunnan Normal University, Kunming, 620092, P.R.China.
}

Abstract: The translation of inheritance nets to default logic has been discussed by Etherington [9], Touretzky [10], etc. Ontologies and inheritance nets are similar in some aspects and based on methods of translating inheritance nets to default logic, a translation of ontologies to default logic with a priority order on defaults is given. Hence, properties of an ontology and the revision of ontologies can be studied in terms of default logic. Ontologies are assumed to be trees under the subsumption relation between concepts and have deduction rules to infer what are not explicitly stated. The statements in ontologies are translated to facts of default theories of the ontologies and the default inheritance of properties are represented by normal defaults with a priority order on them due to the intuition that subclasses overriding superclasses. For an ontology with a tree structure, it is consistent if and only if the default theory of the ontology has a unique extension.

Key words: ontology, default logic, priority order, extension

\section{INTRODUCTION}

Ontologies are important in sharing and reusing knowledge [1,2].An ontology is "an explicit specification of a conceptualisation" [4], where the conceptualisation is an abstract model of some phenomenon in the world through specifying the concepts relevant to that phenomenon.

In an ontology, classes (concepts) consist of individuals: all the individuals of a class have some common properties. A set of relevant concepts and their relationships in an ontology are the conceptualizations [5] 
used to represent the world. Concepts are described by sets of properties true for all the individuals of the class, where the properties are important for the knowledge representation and for distinguishing a concept from the others. Usually properties that are generally true for that concept are also represented. Concepts in an ontology are hierarchically organized through the isa (subsumption) relation to form the ontology's main structure which is further enriched by properties, and by relationships relating concepts.

Our ontology consists of a set of concepts and properties; and three relations: the subsumption relation between concepts; the inheritance and default inheritance relation between concepts and properties. Hence, default logic can be used to study the properties of ontologies. Moreover, in the building and maintaining of ontologies, new information will be added to ontologies continually and ontology revision is necessary. Approaches to belief revision where consistent sets of formulas are changed after adding contradictory information have been treated extensively in the literature [12]. In order to be able to apply the results of belief revision, we need to represent ontologies as sets of formulas. The default theories of ontologies yield such sets. Then based on such sets, we can use the methods of belief revision to study ontology revision. In this paper, we attempt to study the translation of ontologies to default logic.

Similar works $[9,10,11]$ have been investigated by researchers in translating inheritance nets to default logic. Inheritance nets [8] are means for representing taxonomic knowledge. In the inheritance language, there are strict links; default links; and three kinds of objects: categories, individuals and properties. There are some approaches translating inheritance nets to default logic. Etherington and Reiter [9] translated the inheritance nets with exceptions to default logic in which strict links are translated to facts, and defeasible links with exceptions to semi-normal defaults. Unlike Etherington and Reiter's method, in [10], all the links among properties were represented by normal defaults; the links between individuals and properties were represented as facts. A partial order on defaults was defined to decide which inference path was more preferable. In [11], it was assumed that all links were defeasible. Hence, all links were represented uniformly as facts of the corresponding default theory. The defaults only expressed closed world assumption between properties and individuals.

Our ontologies and inheritance nets are similar in that they both consist of two kinds of objects: concepts and properties; and there are strict and defeasible relations among these objects. But they are different. The structure of an inheritance net is a net and there are many connections among concepts and properties. However, an ontology in this paper includes a set of concepts which forms the main structure of the ontology under subsumption relation which is not defeasible. That is, the main structure of an ontology is 
a tree and not a net. Concepts are described by sets of properties, that is, ontologies distinguish concepts from properties. Similar to the default theories of inheritance nets, ontologies can be translated to default logic.

In this paper, we translate all the statements in ontologies to facts and the default inheritance of properties from concepts to their sub-concepts are represented by normal defaults in default logic. There is a priority order on these defaults due to the intuition similar to that of inheritance nets: subclasses override superclasses. Thus, a translation of ontologies to default logic. with priority order on defaults is given. For an ontology with a tree structure under the subsumption relation, the ontology is consistent if and only if the default theory of the ontology has a unique extension.

The paper is organized as follows: in section 2, we give the basic concepts of default logic; the presuppositions and deduction rules for ontologies are discussed in section 3. In section 4, a method of translating ontologies to default logic with priority order on defaults is proposed. We conclude the paper with conclusion and future works.

\section{DEFAULT LOGIC}

Default logic introduced by Reiter $[3,6]$ is one of the most famous formalism for non-monotonic reasoning and its most apparent property is default reasoning produce reliable conclusions but not the irrevocable ones.

Given a first order language $L$, a default rule $\delta$ is an expression of the form $p: q / r$, where $p, q$ and $r$ are formulas in $L$, which are called the prerequisite, consequence and justification of the default. The intuitive explanation of $\delta$ is that if $p$ is provable and it is consistent to assume $q$, then infer $r$. A default theory is a pair $(W, D)$, where $W$ is a set of sentences in $L$, called the facts, and $D$ is a set of defaults. An extension is a maximal consistent set of conclusions that can be drawn from a default theory. We call $\delta$ a normal default if $q=r$ and $(W, D)$ a normal default theory if $D$ is a set of normal defaults.

In a default theory, there are situations that it is reasonable to give some defaults higher priority order than others. We call a default theory $(W, D)$ a prioritized default theory, denoted by $(W, D,<)$, if there is a priority order $<$ on the defaults in $D .(W, D,<)$ is a normal prioritized default theory if $D$ is a set of normal defaults. Then, we give the definition of the extensions for prioritized default theories [7].

Definition 1. Given a prioritized default theory $(W, D,<), \Gamma$ is an operator on sets of sentences such that for any set $S$ of sentences, $\Gamma(S)$ is smallest satisfying the following conditions:

(1) $W \subseteq \Gamma(S)$; 
(2) $T h(\Gamma(S))=\Gamma(S)$, that is, $\Gamma(S)$ is logically closed;

(3) if $p: q / r \in D, p \in \Gamma(S), \neg q \notin S$ and there is no $\delta^{\prime}=p^{\prime}: q^{\prime} / r^{\prime} \in D$ such that $p^{\prime} \in \Gamma(S), \neg q^{\prime} \notin S$ and $\delta^{\prime}<\delta$, then $r \in \Gamma(S)$, where $\delta^{\prime}<\delta$ means that $\delta^{\prime}$ has higher priority than $\delta$.

A set $E$ of sentences is an extension of $(W, D,<)$ iff $\Gamma(E)=E$ for some operator $\Gamma$ satisfying 1,2 and 3 .

Given a prioritized default theory $(W, D,<)$, let $\Gamma$ be an operator defined above and $S$ be a set of sentences, then

(1) a default $\delta=p: q / r \in D$ is priority-free applicable to $\Gamma(S)$ with respect to $S$, if $p \in \Gamma(S)$ and $\neg q \notin S$. For convenience, we say $\delta$ is priorityfree applicable with respect to $S$.

(2) a default $\delta=p: q / r \in D$ is applicable to $\Gamma(S)$ with respect to $S$, if $p \in \Gamma(S)$ and $\neg q \notin S$ and there is no default $\delta^{\prime}=p^{\prime}: q^{\prime} / r^{\prime} \in D$ such that $p^{\prime} \in \Gamma(S), \neg q^{\prime} \notin S$ and $\delta^{\prime}<\delta$. For convenience, we say $\delta$ is applicable with respect to $S$.

Then, we given two propositions about the extensions of normal prioritized default theories.

Proposition 1. Given a normal prioritized default theory $(W, D,<)$, there is at least one extensions.

Proposition 2. Let $E, E^{\prime}$ be two extensions of a normal prioritized default theory $(W, D,<)$, if $E \neq E^{\prime}$, then $E \cup E^{\prime}$ is inconsistent.

For the proof of the above propositions, see [3].

\section{ONTOLOGIES}

In this section, we discuss the presuppositions for ontologies and the deduction rules in ontologies.

The presuppositions about ontology $\boldsymbol{O}$ :

1. The objects in $\boldsymbol{O}$ are classified into four classes: categories, attributes denoted and attribute values. We call category objects concepts denoted by $C, D, \ldots ;$ a combination of attributes and attribute values of the form $[a: v]$ property which represent that the value of attribute $a$ is $v$.

2. There is one kind of relation between concepts: the subsumption relation isa; two kinds of relations between a concept and a property: the inheritance relation prop and the default inheritance relation dprop.

We assume that a concept $C$ has value $v$ at attribute $a$, denoted by $\operatorname{prop}(C,[a: v])$, means that every sub-concept of $C$ has property [a:v], that is, $[a: v]$ is a universal property of $C$; a concept $C$ defautly has value $v$ at attribute $a$, denoted by $\operatorname{dprop}(C,[a: v])$, means that defaultly every subconcept of $C$ has property $[a: v]$, that is, $[a: v]$ is a default property of $C$; 
and $C$ is a sub-concept of $D$, denoted by isa $C, D)$, means that for any property $[a: v]$, if $\operatorname{prop}(D,[a: v])$, then $\operatorname{prop}(C,[a: v])$.

In another words, is a relates two concepts: is $a(C, D)$ ("the dog is an animal", isa(dog, animal) ) and prop relates a concept and a property: $\operatorname{prop}(C,[a: v])$ ("Birds have two legs," prop(bird, [legs:2])); and dprop relates a concept and a property defaultly: $\operatorname{dprop}(C,[a: v])$ ("Cars defaultly have 4 wheels,"dprop (car, [wheels:4] )).

We assume there is only one value for an attribute defined in a concept.

3: For convenience, we assume that all the concepts form a tree under the subsumption relation, that is, if a concept $C$ is subsumed by two concepts $D$ and $F$ then either $D=F$ or one of $D$ and $F$ is subsumed by another, where $D=F$ if and only if $D$ instantiates the same set of properties as $F$ does. Because as a classification of all the individuals in the real world, a tree-like structure is a better choice.

Definition 2. An ontology $\boldsymbol{O}=(U$, isa, prop, dprop, $O)$, where

(1) $U$ is a set of concepts and properties;

(2) isa is a binary relation on concepts in $U$. We assume that isa is a partial order;

(3) prop and dprop are binary relations on concepts and properties in $U$; and

(4) $O$ is a set of statements of the form

$i \operatorname{sa}(C, D) \mid \neg$ isa $(C, D)|\operatorname{prop}(C,[a: v])| \neg \operatorname{prop}(C,[a: v]) \mid \operatorname{dprop}(C,[a: v])$, where $C, D,[a: v] \in U$, and isa, prop, dprop are relations between concepts and between concepts and properties respectively.

In ontologies, all the universal properties of a super-concept are inherited to its sub-concepts. A sub-concept inherits all default properties of a superconcept unless they are overridden in the sub-concepts. That is, for a concept $D$ and its default property $[a: v]$, if $a$ is redefined in a sub-concept $C$ of $D$, then the new statement $\operatorname{prop}\left(C,\left[a: v^{\prime}\right]\right)$ or $\operatorname{dprop}\left(C,\left[a: v^{\prime}\right]\right)$ overrides the statement $\operatorname{dprop}(D,[a: v])$. In this case, the default property $[a: v]$ is not inherited from $D$ to $C$.

With multiple inheritance, there can be conflicting statements, that is, default properties for a same attribute may be declared in more than one super-concepts. In this case, default properties declared in a more specific super-concept is inherited to its sub-concepts, for example, if isa $(C, D)$, $i s a(C, F), i s a(D, F), \operatorname{dprop}(D,[a: v])$, and $\operatorname{dprop}\left(F,\left[a: v^{\prime}\right]\right)\left(\nu \neq v^{\prime}\right)$ and $a$ is not redefined in $C$, then we have $\operatorname{dprop}(C,[a: v])$, that is, $C$ inherits the default property $[a: v]$ from $D$ since $i s a(D, F)$.

Based on what has been discussed above, we give the following deduction rules. Deduction rules in ontologies include inheritance rules, default inheritance rules, transitivity rules and subaltern rules. 
The inheritance rule, if $i s a(C, D)$ and $\operatorname{prop}(D,[a: v])$ are provable, then infer $\operatorname{prop}(C,[a: v])$, is denoted by $i s a(C, D), \operatorname{prop}(D,[a: v]) / \operatorname{prop}(C,[a: v])$; the default inheritance rule, if isa $(C, D)$ and $\operatorname{dprop}(D,[a: v])$ are provable and it is consistent to assume $\operatorname{dprop}(C,[a: v])$, then infer $\operatorname{dprop}(C,[a: v])$, is

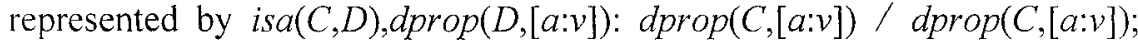
the transitivity rule, if is $a(C, D)$, and is $(D, F)$ are provble, then infer is $a(C, F)$, is represented by is $a(C, D)$, isa $(D, F) / i s a(C, F)$. For the relation between prop and dprop, we have the following subaltern rule: $\operatorname{prop}(C,[a: v]) / \operatorname{dprop}(C,[a: v])$, which means that if a property is a universal property of a concept then it is a default property of the concept.

An ontology has the deduction rules to infer statements not explicitly represented in the ontology.

For the inheritance of default properties, in order to solve the conflicts caused by multiple inheritance, there is a priority order on defaults due to the intuition that sub-concepts overrides super-concepts. That is, give two defaults

$\delta=i s a(C, D), \operatorname{dprop}(D,[a: v]): \operatorname{dprop}(C,[a: v]) / \operatorname{dprop}(C,[a: v])$, and

$\delta^{\prime}=i \operatorname{isa}(C, F), \operatorname{dprop}\left(F,\left[a: v^{\prime}\right]\right): \operatorname{dprop}\left(C,\left[a: v^{\prime}\right]\right) / \operatorname{dprop}\left(C,\left[a: v^{\prime}\right]\right)$, then $\delta<\delta$ ' if is $a(D, F)$ is provable; for example, from the facts isa(tweety, penguin), isa(penguin, ,bird), dprop(penguin,[fly:no]) and dprop(bird,[fly:yes]), we can infer the statement dprop (tweety,[fly:no]).

For the consistency of a set of statements in an ontology or derived from an ontology, we give the following definition:

Definition 3. A set of statements $M$ is consistent if and only if there do not exist concepts $C, D$ and properties $[a: v],\left[a: v^{\prime}\right]$ such that

(1) $i s a(C, D), \neg i s a(C, D) \in T h(M)$;

(2) $\operatorname{prop}(C,[a: v]), \operatorname{prop}\left(C,\left[a: v^{\prime}\right]\right) \in T h(M)$;

(3) $\operatorname{prop}(C,[a: v]), \neg \operatorname{prop}(C,[a: v]) \in T h(M)$;

(4) $\operatorname{dprop}(C,[a: v]), \operatorname{dprop}\left(C,\left[a: v^{\prime}\right]\right) \in T h(M)$;

(5) $\operatorname{prop}(C,[a: v]), \operatorname{dprop}\left(C,\left[a: v^{\prime}\right]\right) \in \operatorname{Th}(M)$; where $v \neq v^{\prime}$, and $\operatorname{Th}(M)$ is the logical closure of $M$, that is, the set of statements inferred from $M$ by the deduction rules.

We say an ontology $\boldsymbol{O}$ is consistent if and only if $O$ is onsistent.

\section{TRANSLATING ONTOLOGIES TO DEFAULT LOGIC}

In this section, we propose the translation of ontologies to default logic, then study the properties of the default theories of ontologies.

Definition 4. Given an ontology $\boldsymbol{O}$, we call a normal prioritized default theory $(O, D,<)$ the default theory of $\boldsymbol{O}$, if 
(1) $D=\{i s a(C, D), \operatorname{dprop}(D,[a: v]): \operatorname{dprop}(C,[a: v]) / d p r o p(C,[a: v]) \mid C$, $D,[a: v] \in U, \operatorname{dprop}(D,[a: v]) \in O\}$;

(2) $<$ is a priority order on the defaults in $D$ such that for any two defaults $\delta_{1}=i s a(C, D), \quad \operatorname{dprop}(D,[a: v]): \operatorname{dprop}(C,[a: v]) / \operatorname{dprop}(C,[a: v])$, $\delta_{2}=i s a(C, F), \operatorname{dprop}\left(F,\left[a: v^{\prime}\right]\right): \operatorname{dprop}\left(C,\left[a: v^{\prime}\right]\right) / \operatorname{dprop}\left(C,\left[a: v^{\prime}\right]\right)$, we have $\delta_{1}<\delta_{2}$, if isa $(D, F)$.

Let $\operatorname{Th}^{+}(O)$ be the first order logical closure of $O$ which is the set of statements inferred from $O$ by the deduction rules except the default inheritance rules.

We say $T^{+}(O)$ is consistent if and only if there do not exist concepts $C, D$, properties $[a: v]$ and $\left[a: v^{\prime}\right]$ such that $(1)$ isa $(C, D), \neg i s a(C, D) \in$ $T h^{+}(O)$; (2) $\operatorname{prop}(C,[a: v]), \operatorname{prop}\left(C,\left[a: v^{\prime}\right]\right) \in \operatorname{Th}^{+}(O) ;(3) \operatorname{prop}(C,[a: v])$, $\neg \operatorname{prop}(C,[a: v]) \in T h^{+}(O) ;(4) \operatorname{dprop}(C,[a: v]), \operatorname{dprop}\left(C,\left[a: v^{\prime}\right]\right) \in T h^{+}(O) ;(5)$ $\operatorname{prop}(C,[a: v]), \operatorname{dprop}\left(C,\left[a: v^{\prime}\right]\right) \in \operatorname{Th}^{+}(O)$.

Definition 5. A statement $\theta$ is a theorem of an ontology $\boldsymbol{O}$, if and only if $\theta$ can be inferred from the deduction rules.

Definition 6. An ontology $\boldsymbol{O}$ is a tree under the subsumption relation isa if there are no concepts $C, D, F \in U$ such that $D \neq F, \neg i s a(D, F)$, $\neg$ isa $(F, D)$, is $a(C, D)$ and isa $(C, F)$.

Using the above propositions, we have the following theorem:

Theorem 1. Given an ontology $\boldsymbol{O}$, if $\boldsymbol{O}$ is a tree and $\operatorname{Th}^{+}(O)$ is consistent then the normal prioritized default theory of the ontology $(O, D,<)$ has a unique extension.

Proof. Since all the defaults in $D$ are normal defaults, the default theory has at least one extensions (Proposition 1). Assume that there are two distinct extensions $E$ and $E^{\prime}$, we prove that $E \cup E^{\prime}$ is consistent. Since $E$ and $E^{\prime}$ are extensions, then $\Gamma(E)=E$ and $\Gamma\left(E^{\prime}\right)=E^{\prime}$. From Definition 3, there are the following five kinds of cases that may make $E \cup E^{\prime}$ inconsistent:

(1) isa $(C, D) \in E, \neg i s a(C, D) \in E^{*}$; in this case, since the derivation of is $a(C, D)$ and $\neg i s a(C, D)$ is not concerned with defaults, then isa $(C, D)$, $\neg i s a(C, D) \in \operatorname{Th}^{+}(O)$ which is impossible since $O$ is consistent.

(2) $\operatorname{prop}(C,[a: v]) \in E, \operatorname{prop}\left(C,\left[a: v^{\prime}\right]\right) \in E^{\prime}$; where $v \neq v^{\prime}$; for the same reason as case one, this case is impossible.

(3) $\operatorname{prop}(C,[a: v]) \in E, \neg \operatorname{prop}(C,[a: v]) \in E$; for the same reason as case one, this case is impossible.

(4) $\operatorname{dprop}(C,[a: v]) \in E, d \operatorname{dprop}\left(C,\left[a: v^{\prime}\right]\right) \in E^{\prime}$; in this case, it is impossible that $\operatorname{dprop}(C,[a: v]), \operatorname{dprop}\left(C,\left[a: v^{\prime}\right]\right) \in \operatorname{Th}^{+}(O)$, since $O$ is consistent; if one of $\operatorname{dprop}(C,[a: v])$ and $\operatorname{dprop}\left(C,\left[a: v^{\prime}\right]\right)$ is in $\operatorname{Th}^{+}(O)$ and the other is derived from some default, then let $\operatorname{dprop}(C,[a: v]) \in T h^{+}(O)$ and there is $\delta=$ isa $(C, D), \operatorname{dprop}\left(D,\left[a: v^{\prime}\right]\right): \operatorname{dprop}\left(C,\left[a: v^{\prime}\right]\right) / \operatorname{dprop}\left(C,\left[a: v^{\prime}\right]\right)$ applicable with respect to $E^{\prime}$. The applicatin of $\delta$ produces $\operatorname{dprop}\left(C,\left[a: v^{\prime}\right]\right) \in E^{\prime}$ which contradicts with $\left.\operatorname{dprop}(C,[a: v]) \in T h^{+}(O)\right) \subseteq E^{\prime}$. If there exists some defaults 
$\delta_{1}$ applicable with respect to $E$ and $\delta_{2}$ applicable with respect to $E$, let $\delta_{1}=\quad i s a(C, D), \operatorname{dprop}(D,[a: v]): \operatorname{dprop}(C,[a: v]) / \operatorname{dprop}(C,[a: v]), \delta_{2}=$ $i s a(C, F), \operatorname{dprop}\left(F,\left[a: v^{\prime}\right]\right): \operatorname{dprop}\left(C,\left[a: v^{\prime}\right]\right) / \operatorname{dprop}\left(C,\left[a: v^{\prime}\right]\right)$, producing $\operatorname{dprop}(C,[a: v]) \in E$ and $\operatorname{dprop}\left(C,\left[a: v^{\prime}\right]\right) \in E^{\prime}$. In the process of constructing extension $E$, before the using of $\delta_{1}, \delta_{1}$ and $\delta_{2}$ are priority-free applicable with respect to $E$ since $i s a(C, D)$, isa $\left.(C, F) \in \operatorname{Th}^{+}(O)\right) \subseteq \Gamma(E)$, dprop $(D,[a: v])$, $\operatorname{dprop}\left(F,\left[a: v^{\prime}\right]\right) \in O \subseteq \Gamma(E)$ and there is no $v_{1}$ such that $v_{1} \neq v, v_{1} \neq v^{\prime}$ and $\operatorname{dprop}\left(C,\left[a: v_{1}\right]\right) \in E$.

Similarly, in the process of constructing $E^{\prime}$, before the using of $\delta_{2}, \delta_{1}$ and $\delta_{2}$ are priority-free applicable with respect to $E$. Hence, if there is no subsumption relation between $D$ and $F$, then $C$ has two parent concepts which contradicts with the assumption that $\boldsymbol{O}$ is a tree. Otherwise, is $a(D, F)$ or is $a(F, D)$, then we have $\delta_{1}<\delta_{2}$, or $\delta_{2}<\delta_{1}$ which means that $\delta_{2}$ is not applicable with respect to $E^{\prime}$ or $\delta_{1}$ is not applicable with respect to $E$ and contradicts with the above assumption.

(5) $\operatorname{prop}(C,[a: v]) \in E, \operatorname{dprop}\left(C,\left[a: v^{\prime}\right]\right) \in E$; in this case, $\operatorname{dprop}(C,[a: v])$ $\in E$ by the subaltern rule, and for the same reason as case (4), this case is impossible.

Hence, $E \cup E^{\prime}$ is consistent and from the contraposition of proposition 2, we have $E=E^{\prime}$ contradictory with our assumption $E$ and $E^{\prime}$ are two distinct extensions of $(O, D,<)$. Therefore, $(O, D,<)$ has only one extension.

The above theorem shows that the set of statements inferred from the default theory of $\boldsymbol{O}$ is consistent if $\boldsymbol{O}$ is a tree under is $a$ and $T h^{+}(O)$ is consistent.

From the definition 4,5 and theorem 1, we have the following proposition: Proposition 3. Given an ontology $\boldsymbol{O}$ with a tree strcuture under is a relation, if $T^{+}(O)$ is consistent, then $E=T h(O)$, where $E$ is the unique extension of the default thory $(O, D,<)$ of $\boldsymbol{O}$.

From theorem 1 and the definitions of consistency of $\boldsymbol{O}$, we have the following proposition:

Proposition 4. Given an ontology $\boldsymbol{O}$ with a tree strcuture under isa relation, $O$ is consistent if and only if $(O, D,<)$ has a unique extension.

\section{CONCLUSION AND FUTURE WORKS}

We provide a method of translating ontologies to default logic with the following properties:

(1) for an ontology $\boldsymbol{O}$, given concepts $C, D, F \in U$, and a property [a:v] $\in U$, if the statements formed from $C, D,[a: v]$ by isa, prop, and dprop relations are in $O$, then they are facts in the default theory of $\boldsymbol{O}$; 
(2) if $\operatorname{dprop}(D,[a: v]) \in O$, then the default rule isa $(C, D), \operatorname{dprop}(D,[a: v])$ : $\operatorname{dprop}(C,[a: v]) / \operatorname{dprop}(C,[a: v])$ is in $D$; the priority order < on the defaults of $D$ is due to the intuition that sub-concepts overrides superconcepts.

(3) for an ontology $\boldsymbol{O}$ with tree structure under isa relation, $\boldsymbol{O}$ is consistent if and only $(O, D,<)$ has exactly one extension.

Future works includes: the study of the properties of ontologies in terms of default logic, the revision of default theories and ontology revision based on the default theories of ontologies.

\section{NOTES}

The work is partially supported by the National NSF of China (No. 60373042, 60273019 and 60037017), the NSF of Yunnan Province( No. 2004F0017Q), and the Education Bureau Youth Fund of Yunnan Province (No. 03Y312D and 04Y769D).

\section{REFERENCES}

[1] ValentinaTamma, A. and Trevor, J. SupportingInheritance Mechanisms in Ontology Representation. In Proc. of the 12th International Conference on Knowledge Engineering and Knowledge Management, pages 140-155, 2000.

[2] Guarino, N. and Welty, C. Ontological Analysis of Taxonomic Relationship. In Proc. of International Conference on Conceptual Modelling, pages 210-224, 2000.

[3] Besnard, P. An introduction to default logic, Springer-Verlag, Berlin, 1989.

14] Gruber,T. A Translation Approach to Portable Ontology Specifications. In Knowledge Acquisition, 5(2):199-220, 1993.

[5] Genesereth, M. R., Nillson, N. LogicalFoundations of Artificial Intelligence, Morgan Kauffman, 1987.

[6] Reiter, R. A Logic for Default Reasoning. In Artificial Intelligence, 13:81-132, 1980.

[7] Antoniou, G. Priorities in Default Logic Revisited. In Proc. of 4th Congress of the Italian Association for Artificial Intelligence, Springer, pages 152-162,1995.

[8] Touretzky, D. S. The Mathematics of InheritanceSystems, Morgan Kaufmann, Pitman, London, 1986.

[9] Etherington, D. Reiter, R. On Inheritance Hierarchies with Exception. In Proc. of AAAI83, William Kaufman, Los Altos, CA, pages 104-108, 1983.

[10] Touretzky, D. Implicit Ordering of Defaults in Inheritance systems. In Readings in Nonmonotonic Reasoning, Morgan Kaufmann, Los Altos, CA, pages 106-109, 1987.

[11] Ingrid, N. Translating Inheritance Nets to Default Logic, http://www.ubka.unikarlsruhe.de/cgi-bin/psounzip/ira/1994/25/25.pdf, 1994.

[12] Bochman, A. A foundational theory of belief and belief change. In Artificial Intelligence, 108(1-2):309-352, 1999. 